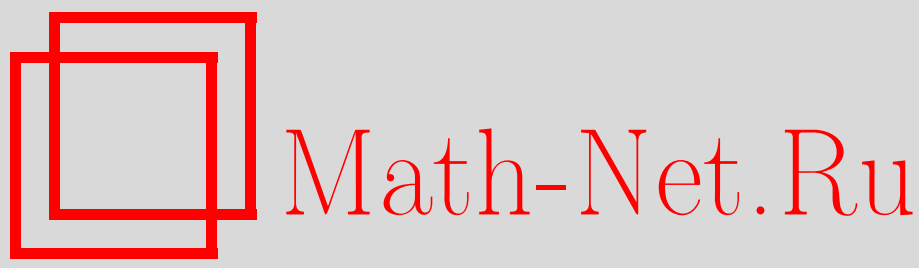

А. П. Старовойтов, Н. А. Старовойтова, Асимптотика определителей Адамара и поведение строк таблиц Паде и Чебышёва для суммы экспонент, Матем. сб., 1996, том 187, номер 2, 141-157

DOI: https://doi.org/10.4213/sm113

Использование Общероссийского математического портала Math-Net.Ru подразумевает, что вы прочитали и согласны с пользовательским соглашением

http://www.mathnet.ru/rus/agreement

Параметры загрузки:

IP : 54.147 .182 .235

26 апреля 2023 г., 15:22:11 
УДК 517.53

\author{
А.П. Старовойтов, Н.А. Старовойтова
}

\title{
Асимптотика определителей Адамара и поведение строк таблиц Паде и Чебышёва для суммы экспонент
}

Для функции $f(z)=\sum_{j=1}^{k} e^{\lambda_{j} z}$ установлены асимптотические равенства для определителей Адамара ее коэффициентов Тейлора. Это позволило в некоторых случаях найти асимптотику уклонения от $f(z)$ аппроксимаций Паде $\Pi_{n, m}(z)$ и рациональных функций наилучшего равномерного приближения $r_{n, m}^{*}(z)=p_{n}^{*}(z)=q_{m}^{*}(z)$ при фиксированном $m$ и $n \rightarrow \infty$.

Библиография: 24 названия.

\section{§1. Введение}

Пусть $\mathbf{R}_{n, m}$ - класс всех рациональных функций, числитель которых имеет степень не вьше $n$, а знаменатель - не выше $m$. Для каждой аналитической в области $G \supset \bar{D}=\{z:|z| \leqslant 1\}$ функции $f(z)$ соотношение

$$
\left(Q_{m, n} \cdot f-P_{n, m}\right)(z)=A z^{n+m+1}+\cdots
$$

единственным образом определяет рациональную функцию

$$
\Pi_{n, m}(z)=\Pi_{n, m}(z ; f)=\frac{P_{n, m}(z)}{Q_{m, n}(z)}
$$

из $\mathbf{R}_{n, m}$, которая называется $(n, m)$-й аппроксимацией Паде. Следующую таблицу

$$
\left[\begin{array}{ccccc}
\Pi_{0,0} & \Pi_{1,0} & \ldots & \Pi_{n, 0} & \ldots \\
\Pi_{0,1} & \Pi_{1,1} & \ldots & \Pi_{n, 1} & \ldots \\
\ldots \ldots & \ldots & \ldots & \ldots \\
\Pi_{0, m} & \Pi_{1, m} & \ldots & \Pi_{n, m} & \ldots \\
\ldots \ldots & \ldots \ldots \ldots & \ldots & \ldots \ldots \ldots
\end{array}\right]
$$

при этом называют таблицей Паде функции $f(z)$. Наряду с таблицей (1) будем рассматривать и таблицу Чебышёва, полученную из (1) заменой функции $\Pi_{n, m}(z)$ на $r_{n, m}^{*}(z)$, где $r_{n, m}^{*}(z)$ - одна из дробей наилучшего равномерного приближения $f(z)$ в классе $\mathbf{R}_{n, m}$. Последняя определяется из соотношений

$$
\mathbf{R}_{n, m}(f, \bar{D})=\inf _{r \in \mathbf{R}_{n, m}}\|f-r\|=\left\|f-r_{n, m}^{*}\right\|
$$

где $\|g\|=\sup \{|g(z)|: z \in \bar{D}\}$. 
Функции $\Pi_{n, m}(z ; f)$ введены Паде в 1892 году при исследовании непрерывных дробей $\exp z$ (см. [1], [2]). В частности, для экспоненты им был найден явный вид $\Pi_{n, m}(z)$ и доказана равномерная сходимость на компактах в $\mathbb{C}$ основных элементов таблицы (1) (строк, столбцов и диагонали). Позже (1913 г.) Перрон (см. [3]) получил компактное интегральное представление числителя и знаменателя дроби $\Pi_{n, m}\left(z ; e^{z}\right)$, что позволило (см [4]) установить асимптотику главной диагонали (1).

В работе [5] В.К. Дзядык и Л.И. Филозоф предложили другой метод исследования поведения диагонали таблицы Паде (аппроксимационный метод) и показали, что в смысле сходимости к функции ехр $z$ диагонали таблиц Паде и Чебышёва ведут себя одинаково. Поведение строк таблиц Паде и Чебышёва исследовалось Саффом в [6]-[8]. В этих работах, в частности, найдена асимптотика для разности $e^{z}-r_{n, m}^{*}(z)$ при фиксированном $m, n \rightarrow \infty$ и $z \in \bar{D}$.

Вопросы поведения элементов таблиц Паде и Чебышёва на различных множествах из $\mathbb{C}$ рассматривались в работах Саффа, Варги и других математиков (по этому поводу см., например, [9]). Не останавливаясь подробно на результатах, полученных в этом направлении, отметим лишш известную задачу о скорости рациональной аппроксимации функции $e^{-x}$ на полуоси $[0,+\infty)$. Как известно, ее полное решение было найдено в 1987 году А. А. Гончаром и Е. А. Рахмановым на основе конструкции многоточечных аппроксимаций Паде [10]. Работе [10] предшествовали многочисленные публикации так или иначе связанные с этой задачей. В них, в частности, имеется как обилие предлагаемых методов исследования, так и обширная библиография, относящаяся к рациональной аппроксимации ехр $z$ и ее аппроксимациям Паде (см., например, [11]-[13]). Подробньй обзор этих результатов имеется также и в цитируемой выше работе А. А. Гончара и Е. А. Рахманова [10].

Вернемся опять к таблице (1), когда $z \in \bar{D}$. В этом случае таблица Паде экспоненты полностью исследована Д. Браессом в [14]: для любого $z \in \bar{D}$ и $n+m \rightarrow \infty$

$$
e^{z}-\Pi_{n, m}\left(z ; e^{z}\right)=\frac{(-1)^{m} n ! m ! e^{\frac{2 m}{n+m} z}}{(n+m) !(n+m+1) !} z^{n+m+1}(1+o(1))
$$

Аналогичный результат, относяшийся к таблице Чебышёва, получен Л. Трефезеном [15]: при $n+m \rightarrow \infty$

$$
\left\|e^{z}-r_{n, m}^{*}(z)\right\|=\frac{m ! n !}{(n+m) !(n+m+1) !}(1+o(1)) .
$$

Основываясь на своем результате для круга, Д. Браесс исследовал и случай отрезка $[-1 ; 1]$ : при $n+m \rightarrow \infty$

$$
\mathbf{R}_{n, m}\left(e^{x} ;[-1 ; 1]\right)=\frac{m ! n !}{2^{n+m}(n+m) !(n+m+1) !}(1+o(1)) .
$$

Из полученных результатов можно сделать вывод о том, что в смысле сходимости к $\exp z$ в $\bar{D}$, поведение отдельных элементов (строк, столбцов, диагонали и др.) таблищ Паде и Чебьшёва одинаково в смысле порядка сходимости. С другой стороны, к настояшему времени экспонента остается единственной функцией, для которой 
рассматриваемые таблицы изучены в полном объеме. В связи с этим А.И. Аптекаревым в [16] подробно рассматривается задача (постановка принадлежит Е. М. Никишину; по этому поводу см. [16]) о сходимости аппроксимаций Паде для суммы экспонент. Сформулируем основной результат этой работы.

Teоpema [16]. Пусть

$$
f(z)=\sum_{j=1}^{k} e^{\lambda_{j} z},
$$

где $\lambda_{j}(j=1,2, \ldots, k)$ - различные комплексные числа; $\left|\lambda_{1}\right|=\left|\lambda_{2}\right|=\cdots=$ $\left|\lambda_{s}\right|>\left|\lambda_{s+1}\right|>\cdots>\left|\lambda_{k}\right|$. Тогда если $s=1$, то при любом фиксированном $m=0,1,2, \ldots \Pi_{n, m}(z ; f)$ равномерно сходится на $\bar{D} \kappa f(z) ;$ если $s \geqslant 2$, то такая сходимость имеет место при любом фиксированном $m=p s$, $p=0,1,2, \ldots$.

Доказательство теоремы [16] основано на полученной асимптотике определителей Адамара и приводится с помощью одной леммы А. А. Гончара [17, лемма 1].

В данной работе установлены асимптотические равенства для определителей Адамара, уточняющие результаты из [16]. Это позволило в некоторых случаях найти скорость сходимости строк таблиц Паде и Чебышёва для суммы экспонент. В частности, установлена асимптотика уклонения $\Pi_{n, m}(z)$ и $r_{n, m}^{*}(z)$ от $f(z)$ при фиксированном $m$ и $n \rightarrow \infty$. Основные результаты статьи докладывались на Одесской школе по теории функций (1991 г.) и анонсированы в [18].

Авторы благодарят профессора В.Н. Русака (Белорусский государственньй университет) и профессора А.А. Пекарского (Гроденский государственный университет) за полезные обсуждения и помощь в работе.

\section{§2. Основные теоремы}

Перейдем к формулировкам полученных результатов.

Теорема 1. Пусть функиия $f(z)$ определена соотношением (4) и $s=1$. Тогда для любого фиксированного $m$ и $z \in \bar{D}$ при $n \rightarrow \infty$ справедливо следующее асимптотическое равенство

$$
f(z)-\Pi_{n, m}(z ; f)=\frac{(-1)^{m} m !\left(\lambda_{1} z\right)^{n+m+1}}{n^{2 m}(n+1) !}\left(1+O\left(\frac{1}{n}\right)\right) .
$$

ТЕорема 2. В условиях теоремы 1 при любом фиксированном $m$ и $n \rightarrow \infty$ имеет место асимптотическое равенство

$$
\begin{aligned}
\mathbf{R}_{n, m}(f ; \bar{D}) & =\left\|f-\Pi_{n, m}\right\|\left(1+O\left(\frac{1}{n}\right)\right) \\
& =\frac{m !\left|\lambda_{1}\right|^{n+m+1}}{n^{2 m}(n+m) !}\left(1+O\left(\frac{1}{n}\right)\right) .
\end{aligned}
$$

Сформулированные теоремы относятся к случаю, ког да в сумме (4) есть одна доминируюшая компонента. Как следует из $(2),(3),(5)$ и (6), в этой ситуации строки таблиц Паде и Чебьшёва ведут себя в смысле сходимости к $f(z)$ одинаково. При этом основной вклад в асимптотику вносит та компонента, которая доминирует. 
ТЕОРема 3. Пусть функиия $f(z)$ определена соотношением (4), где $k=s$, $\lambda_{j}=R \exp \left\{\left(\varphi_{0}+2 \pi j / s\right) i\right\} \quad(j=0,1, \ldots, s-1), \varphi_{0} \in[0,2 \pi)$. Тогда при любом фиксированном $m$ и $n \rightarrow \infty$ справедливо асимптотическое равенство

$$
f(z)-\Pi_{s n, s m}(z ; f)=\frac{\left(-s^{2} R^{s} e^{i s \varphi_{0}}\right)^{m} m ! s}{(n s)^{m(s+1)}(n s+s) !} z^{s(n+m+1)}\left(1+O\left(\frac{1}{n}\right)\right) .
$$

ТЕОРЕма 4. В предположениях теоремь 3 при любом фиксированном $m$ и $n \rightarrow \infty$ справедливо асимптотическое равенство

$$
\begin{aligned}
\mathbf{R}_{s n, s m}(f ; \bar{D}) & =\left\|f-\Pi_{n, m}\right\|\left(1+O\left(\frac{1}{n}\right)\right) \\
& =\frac{s^{2 m} R^{s m} m ! s}{(n s)^{m(s+1)}(n s+s) !}\left(1+O\left(\frac{1}{n}\right)\right) .
\end{aligned}
$$

В теоремах 3, 4 сумма экспонент имеет $s$ доминирующих компонент. Полагая $R=1, \varphi_{0}=\pi / 2\left(\varphi_{0}=0\right), s=k=2$, получим, что $f(z)=2 \cos z(f(z)=2 \operatorname{ch} z)$, и в силу однородности оператора Паде $\Pi: f \rightarrow \Pi_{n, m}$ равенства $(7),(8)$ дают описание поведения строк таблиц Паде и Чебышёва функций $\cos z, \operatorname{ch} z$, которое согласуется с полученным ранее в работах [19], [20]. Выбор чисел в двух предыдущих теоремах обусловлен, прежде всего, этими примерами. Как будет видно из доказательства теорем 1-4 (см. также [16]) поведение строк таблицы Паде зависит от алгебраических свойств чисел $\left(\lambda_{j}\right)_{j=1}^{k}$. При этом аналоги теорем 3,4 можно получить, зная асимптотику изменения величин $\lambda_{1}^{n}+\lambda_{2}^{n}+\cdots+\lambda_{k}^{n}$ при $n \rightarrow \infty$. Без учета этого обстоятельства удается получить лишь оценку сверху.

Теорема 5. Пусть функиия $f(z)$ определена равенством (4) $u\left|\lambda_{1}\right|=\cdots=$ $\left|\lambda_{s}\right|=1>\left|\lambda_{s+1}\right| \geqslant \cdots \geqslant\left|\lambda_{k}\right|$. Тогда при любом фиксированном $m=p s$, $p=0,1,2, \ldots$, и достаточно больиих $n$ справедливо неравенство

$$
\mathbf{R}_{n, m}(f ; \bar{D}) \leqslant\left\|f-S_{n, m}\right\| \leqslant \frac{c(m, s)}{(n+1) ! n^{m}}
$$

где $S_{n, m}(\cdot ; f) \in \mathbf{R}_{n, m}$ - частные рациональные суммы Фурье, знаменатель которых совпадает со знаменателем дроби Паде $\Pi_{n, m}(z ; f)$.

В связи с последней теоремой заметим, что с точки зрения рациональной аппроксимации со свободньми полюсами рациональные суммы Фурье обстоятельно изучались в работах В.Н. Русака (см., например, [20], [24]). В частности, в [20] рассмотрена аппроксимация такими суммами некоторых аналитических функций $f(z)$, когда в качестве полюсов $S_{n, m}(z ; f)$ выбраны полюса $\Pi_{n, m}(z ; f)$ (определение $S_{n, m}(z ; f)$ см. далее).

\section{§3. Доказательства теорем 1, 2}

Приступим к доказательству теорем 1,2 . Пусть

$$
f(z)=\sum_{n=0}^{\infty} c_{n} z^{n}
$$


- разложение в ряд Тейлора функции $f(z)$. Введем в рассмотрение определители Адамара $\left(c_{j}=0\right.$ при $\left.j<0\right)$

$$
\Delta_{n, m}=\left|\begin{array}{cccc}
c_{n-m+1} & c_{n-m+2} & \ldots & c_{n} \\
c_{n-m+2} & c_{n-m+3} & \ldots & c_{n+1} \\
\ldots \ldots \ldots \ldots \ldots \ldots \ldots \ldots & \ldots \ldots \ldots \ldots \\
c_{n} & c_{n+1} & \ldots & c_{n+m-1}
\end{array}\right|
$$

ЛЕмма 1. Пусть $\left(\lambda_{j}\right)_{j=1}^{k}$ - набор комплексных чисел $\left|\lambda_{1}\right|>\left|\lambda_{2}\right| \geqslant\left|\lambda_{3}\right| \geqslant$ $\cdots \geqslant\left|\lambda_{k}\right|, f(z)=\sum_{j=1}^{k} e^{\lambda_{j} z}$. Тогда для любого фиксированного $\mathrm{m}$ и $n \rightarrow \infty$

$$
\Delta_{n, m}=\frac{\left(-\lambda_{1}\right)^{m(m-1) / 2}}{n^{m(m-1)}} \prod_{j=1}^{m}(m-j) ! c_{n-m+j}\left(1+O\left(\frac{1}{n}\right)\right) .
$$

ДокАЗАТЕЛЬСТво. Для функции $f(z)$ коэффициенты степенного ряда (10) имеют вид

$$
c_{n}=\frac{1}{n !} \sum_{j=1}^{k} \lambda_{j}^{n}
$$

Поэтому

$$
\frac{c_{n+1}}{c_{n}}=\frac{1}{n+1} \frac{\sum_{j=1}^{k} \lambda_{j}^{n+1}}{\sum_{j=1}^{k} \lambda_{j}^{n}}=\frac{\lambda_{1}}{n+1}\left(1+O\left(q^{n}\right)\right),
$$

где $q$ можно считать равньм $\lambda_{2} / \lambda_{1}$, т.е. $|q|<1$. Тогда, разделив $j$-столбец определителя $\Delta_{n, m}$ на $c_{n-m+j}(j=1,2, \ldots, m)$, получим

$$
\Delta_{n, m}=\lambda_{1}^{m(m-1) / 2} \prod_{j=1}^{m} c_{n-m+j}\left|\begin{array}{ccc}
1 & \ldots & 1 \\
l_{1}(n-m+1) & \ldots & l_{1}(n) \\
l_{2}(n-m+1) & \ldots & l_{2}(n) \\
l_{m-1}(n-m+1) & \ldots & l_{m-1}(n)
\end{array}\right|,
$$

где $l_{i}(j)=c_{j+i} /\left(\lambda_{1}^{i} c_{j}\right), 1 \leqslant i \leqslant m-1, n-m+1 \leqslant j \leqslant n$. С учетом (13) имеем

$$
\begin{aligned}
l_{i}(j) & =\frac{1}{\lambda_{1}^{i}} \frac{c_{j+1}}{c_{j}} \frac{c_{j+2}}{c_{j+1}} \cdots \frac{c_{j+i}}{c_{j+i-1}} \\
& =\frac{1}{(j+1)(j+2) \cdots(j+i)}\left(1+O\left(q^{n}\right)\right) .
\end{aligned}
$$

Определитель в (14) имеет фиксированный порядок $m$ и по определению равен сумме конечного числа слагаемых, определяемого числом всех перестановок чисел $1,2, \ldots, m$. Каждое такое слагаемое, содержащее множитель $O\left(q^{n}\right)$, имеет более высокий порядок убывания (порядок геометрической прогрессии), чем те слагаемые, в которых этот множитель отсутствует. Поэтому главньй член асимптотики $\Delta_{n, m}$ зависит только от первых слагаемых в представлении (15) величин $l_{i}(j)$. Следовательно,

$$
\Delta_{n, m}=\lambda_{1}^{m(m-1) / 2} H(n, m) \prod_{j=1}^{m} c_{n-m+j}\left(1+O\left(q^{n}\right)\right),
$$


где

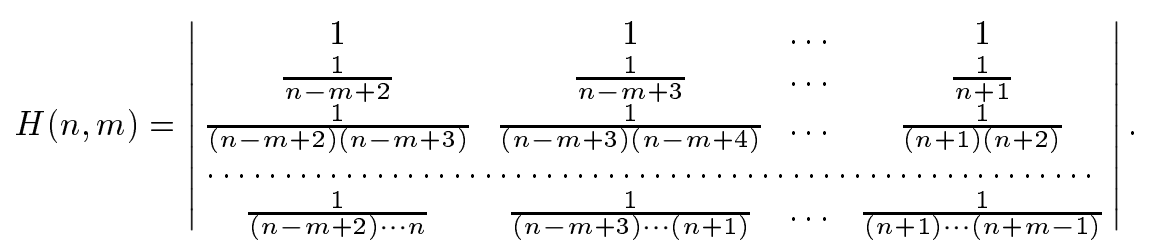

Займемся вычислением определителя $H(n, m)$. Для этого разделим каждый столбец этого определителя на элемент этого определителя, стоящий в последней $m$-ой строке. В результате получим

$$
H(n, m)=\prod_{i=1}^{m} \frac{1}{\prod_{j=1}^{m-1}(n-m+j+i)} K(n, m),
$$

где

$$
K(n, m)=\left|\begin{array}{cccc}
(n-m+2)_{(m-1)} & (n-m+3)_{(m-1)} & \ldots & (n+1)_{(m-1)} \\
(n-m+3)_{(m-2)} & (n-m+4)_{(m-2)} & \ldots & (n+2)_{(m-2)} \\
(n-m+4)_{(m-3)} & (n-m+5)_{(m-3)} & \ldots & (n+3)_{(m-3)} \\
\ldots \ldots \ldots \ldots \ldots \ldots \ldots \ldots \ldots \ldots \ldots \ldots \ldots \ldots \ldots \ldots \ldots \ldots \ldots \ldots \ldots \ldots \ldots \ldots \ldots \ldots & \ldots \ldots \ldots \ldots
\end{array}\right|,
$$

$\mathrm{a}(j)_{(i)}=j(j+1) \cdots(j+i-1)$. Теперь, учитывая, что

$$
(j)_{(i)}-(j+1)_{(i)}=-i(j+1)_{(i-1)},
$$

и вычитая последовательно из первого столбца определителя (18) второй столбец, из второго - третий и т.д., получим

$$
K(n, m)=(-1)^{m-1}(m-1) ! K(n, m-1) .
$$

Так как $K(n, 2)=-1$, отсюда и из (17) окончательно будем иметь

$$
H(n, m)=(-1)^{m(m-1) / 2} \prod_{j=1}^{m-1} j ! \prod_{i=1}^{m} \frac{1}{\prod_{j=1}^{m-1}(n-m+j+i)}
$$

Учитывая (20) и (16), приходим к равенству (12). Лемма 1 доказана.

Для знаменателя аппроксимации Паде $\Pi_{n, m}(z)=P_{n, m}(z) / Q_{m, n}(z)$ имеет место представление (см. [9, с. 16])

$$
Q_{m, n}(z)=\left|\begin{array}{ccccc}
c_{n-m+1} & c_{n-m+2} & \ldots & c_{n} & c_{n+1} \\
c_{n-m+2} & c_{n-m+3} & \ldots & c_{n+1} & c_{n+2} \\
\ldots \ldots \ldots \ldots \ldots \ldots \ldots \ldots \ldots \ldots \ldots \ldots \ldots \ldots \ldots \ldots & \ldots \ldots \ldots & \ldots \ldots & \ldots \ldots \\
c_{n} & c_{n+1} & \ldots & c_{n+m-1} & c_{n+m} \\
z^{m} & z^{m-1} & \ldots & z & 1
\end{array}\right|
$$

Определитель $\Delta_{n, m}^{0} \stackrel{\text { def }}{=} \Delta_{n, m}$ является минором элемента 1 в последней строке определителя (21). Обозначим через $\Delta_{n, m}^{j}$ миноры элементов $z^{j}(j=0,1, \ldots, m)$ из этой строки. 
Лемма 2. Пусть $\left(\lambda_{j}\right)_{j=1}^{k}$ - набор комплексньх чисел $\left|\lambda_{1}\right|>\left|\lambda_{2}\right| \geqslant\left|\lambda_{3}\right| \geqslant$ $\cdots \geqslant\left|\lambda_{k}\right|, f(z)=\sum_{j=1}^{k} e^{\lambda_{j} z}$. Тогда для любого фиксированного $\mathrm{m}$ и $n \rightarrow \infty$ справедливо асимптотическое равенство

$$
\Delta_{n, m}^{j}=\frac{\left(-\lambda_{1}\right)^{m(m-1) / 2} g(m)}{n^{m(m-1)}} \prod_{j=1}^{m-1} j ! c_{n+1} \prod_{\substack{k=1 \\ k \neq j}}^{m} c_{n-m+k}(1+O(1 / n))
$$

где $g(m)$ зависит только от $m, 1 \leqslant j \leqslant m$.

ДоКАЗАТЕЛЬСТво. Проведя преобразования, аналогичные произведенньм при вычислении определителя $\Delta_{n, m}$, будем иметь

$$
\Delta_{n, m}^{j}=\lambda_{1}^{\frac{m(m-1)}{2}} c_{n+1} \prod_{\substack{k=1 \\ k \neq j}}^{m} c_{n-m+j} H^{j}(n, m)\left(1+O\left(q^{n}\right)\right)
$$

где

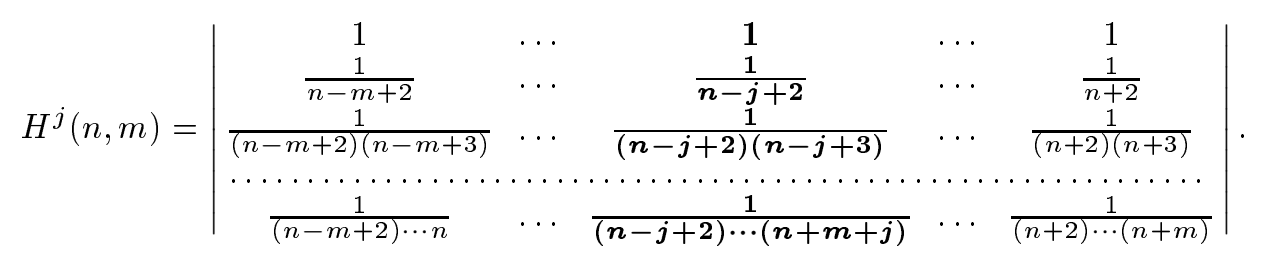

Полужирньм шрифтом отмечен $(m-j+1)$-й столбец, которьй опускается. Разделив каждый столбец на элемент из этого столбца, стоящий в последней $m$-ой строке, получим

$$
H^{j}(n, m)=\prod_{\substack{i=1 \\ i \neq m-j+1}}^{m+1} \frac{1}{\prod_{k=1}^{m-1}(n-m+k+i)} K^{j}(n, m),
$$

где

$$
\begin{aligned}
& K^{j}(n, m)=
\end{aligned}
$$

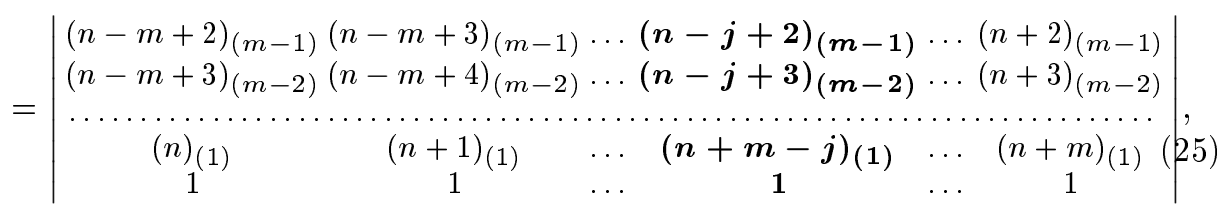

$j=1,2, \ldots, m$. Покажем, что

$$
K^{j}(n, m)=(-1)^{\frac{m(m-1)}{2}} \prod_{j=1}^{m-1} j ! g(m)
$$


где $g(m)$ зависит только от $m$. Действительно, из определения $K^{j}(n, m)$ и $K(n, m)$

$$
\begin{aligned}
K^{0}(n, l) & =K(n, l), & l & =2, \ldots, m ; \\
K^{l}(n, l) & =K(n+1, l), & l & =2, \ldots, m .
\end{aligned}
$$

Выгтем последовательно из первого столбца определителя (25) второй столбец, из второго - третий и т.д. При этом для получаемых разностей будем применять формулы (19) для всех столбцов за исключением $(m-j)$-го. Каждый элемент $(m-j)$-го столбца перепишем в виде

$$
\begin{aligned}
& (n-j+1)_{(m-1)}-(n-j+3)_{(m-1)}=\left\{(n-j+1)_{(m-1)}-(n-j+2)_{(m-1)}\right\} \\
& +\left\{(n-j+2)_{(m-1)}-(n-j+3)_{(m-1)}\right\}, \\
& (n-j+2)_{(m-2)}-(n-j+4)_{(m-2)}=\left\{(n-j+2)_{(m-2)}-(n-j+3)_{(m-2)}\right\} \\
& +\left\{(n-j+3)_{(m-2)}-(n-j+4)_{(m-2)}\right\}, \\
& (n+m-j-1)_{(1)}-(n+m-j+1)_{(1)}=\{-1\}+\{-1\},
\end{aligned}
$$

а затем применим формулы (19). В результате получим

$$
K^{j}(n, m)=(-1)^{m-1}(m-1) !\left[K^{j-1}(n, m-1)+K^{j}(n, m-1)\right] .
$$

Из (27), (28) с учетом величины определителя $K(n, m)$ легко выводится формула (26). Учитывая (26), (24), (23), получаем равенство (22). Лемма доказана.

СлеДСТвИЕ. При условии лемм 1, 2

$$
Q_{m, n}(z)=\Delta_{n, m}(1+O(1 / n)) .
$$

ДОКАЗАТЕЛЬСТво. Из (21) следует, что

$$
Q_{m, n}(z)=\Delta_{n, m} \sum_{j=0}^{m} \frac{\Delta_{n, m}^{j}}{\Delta_{n, m}} z^{j} .
$$

Отсюда и из равенств (12), (22) получим (29).

Введем в рассмотрение определители $(m+1)$-го порядка

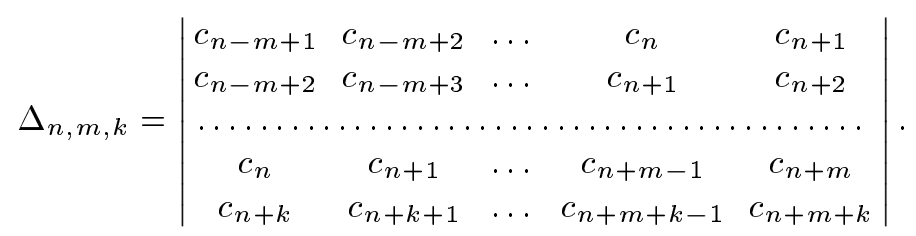

ЛЕмма 3. При условии лемм 1, 2 для любого фиксированного $m$ и $n \rightarrow \infty$

$$
\Delta_{n, m, k}=\frac{\left(-\lambda_{1}\right)^{m(m+1) / 2} \lambda_{1}^{k-1}}{n^{m(m+1)+k-1}} \prod_{j=1}^{m}(m-j) !(j+k-1) \prod_{j=1}^{m+1} c_{n-m+j}(1+O(1 / n)) .
$$


ДОКАЗАТЕЛЬСТво. При $k=1 \Delta_{n, m, 1}=\Delta_{n+1, m+1}$ и (30) следует из (12). Пусть $k \geqslant 2$. Проделав те же преобразования с определителем $\Delta_{n, m, k}$, что и в леммах 1,2 , будем иметь

$$
\begin{aligned}
& \Delta_{n, m, k}=\lambda_{1}^{m(m+1) / 2+k-1} \prod_{j=1}^{m+1} c_{n-m+j} \\
& \times\left|\begin{array}{cccc}
1 & \ldots & 1 & 1 \\
l_{1}(n-m+1) & \ldots & l_{1}(n) & l_{1}(n+1) \\
l_{2}(n-m+1) & \ldots & l_{2}(n) & l_{2}(n+1) \\
\ldots \ldots \ldots \ldots \ldots \ldots \ldots \ldots \ldots \ldots \ldots \ldots \ldots \ldots \ldots \ldots \ldots \ldots \ldots \\
l_{m-1}(n-m+1) & \ldots & l_{m-1}(n) & l_{m-1}(n+1) \\
l_{m+k-1}(n-m+1) & \ldots & l_{m+k-1}(n) & l_{m+k-1}(n+1)
\end{array}\right| .
\end{aligned}
$$

Учитывая представление величин $l_{i}(j)$ в виде $(15)$ и равенства

$$
l_{m+k-1}(j)=\frac{1}{(j+1)(j+2) \cdots(j+m+k-1)}\left(1+O\left(q^{n}\right)\right),
$$

при $n-m+1 \leqslant j \leqslant n+1$ с помощью уже известных рассуждений получим

$$
\Delta_{n, m, k}=\lambda_{1}^{m(m+1) / 2+k-1} \prod_{j=1}^{m+1} c_{n-m+j} H(n, m, k)\left(1+O\left(q^{n}\right)\right),
$$

где

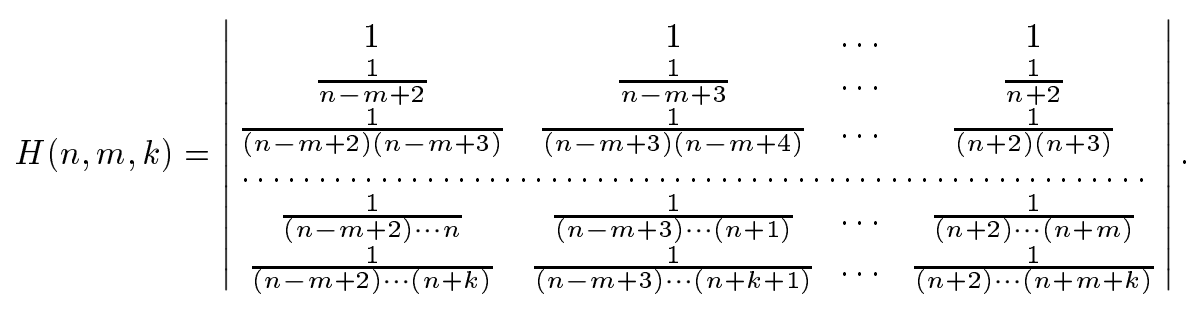

Вынося в каждом столбце элемент последней строки, будем иметь

$$
H(n, m, k)=\prod_{i=1}^{m+1} \frac{1}{\prod_{j=1}^{m+k-1}(n-m+j+i)} K(n, m, k),
$$

где

$$
K(n, m, k)=\left|\begin{array}{cccc}
(n-m+2)_{(m+k-1)} & (n-m+3)_{(m+k-1)} & \ldots & (n+2)_{(m+k-1)} \\
(n-m+3)_{(m+k-2)} & (n-m+4)_{(m+k-2)} & \ldots & (n+3)_{(m+k-2)} \\
\ldots \ldots \ldots \ldots \ldots \ldots \ldots \ldots \ldots \ldots \ldots \ldots \ldots \ldots \ldots \ldots \ldots \ldots \ldots \ldots \ldots \ldots \ldots \ldots \ldots \ldots \ldots \ldots \\
(n+1)_{(k)} & (n+2)_{(k)} & \ldots & (n+m+1)_{(k)} \\
1 & 1 & \ldots & 1
\end{array}\right| .
$$


В определителе $K(n, m, k)$ вычтем из первого столбца второй столбец, из второго - третий и т. д., а затем в полученном определителе каждый столбец разделим на элемент этого столбца, стоящий в последней строке. В результате получим

$$
K(n, m, k)=(-1)^{m} \prod_{j=1}^{m}(j+k-1)(n+j+1)_{(k-1)} K(n+1, m) .
$$

Учитывая значение определителя $K(n+1, m)$, окончательно получим

$$
K(n, m, k)=(-1)^{m(m+1) / 2} \prod_{j=1}^{m}(m-j) !(j+k-1)(n+j+1)_{(k-1)} .
$$

Теперь из (33), (32) и (31) легко получить равенство (30). Лемма 3 доказана.

ДокАЗАТЕЛЬСТВо ТЕОРЕМЫ 1 . Пусть $\Pi_{n, m}(z)=P_{n, m}(z) / Q_{m, n}(z)-$ аппроксимации Паде функции

$$
f(z)=\sum_{j=1}^{k} e^{\lambda_{j} z}
$$

и $\left|\lambda_{1}\right|>\left|\lambda_{2}\right| \geqslant\left|\lambda_{3}\right| \geqslant \cdots \geqslant\left|\lambda_{k}\right|$. Положим $R_{n, m}(z)=Q_{m, n}(z) f(z)-P_{n, m}(z)$. Тогда $[9$, с. 17$]$

$$
R_{n, m}(z)=\sum_{k=1}^{\infty} \Delta_{n, m, k} z^{n+m+k}=\Delta_{n, m, 1} z^{n+m+1}+L(z) .
$$

Для оценки $L(z)$ воспользуемся леммой 3: для любого $z \in \bar{D}$

$$
|L(z)| \leqslant \sum_{k=2}^{\infty}\left|\Delta_{n, m, k}\right|=\left|\Delta_{n, m, 1}\right| \sum_{k=2}^{\infty}\left|\frac{\Delta_{n, m, k}}{\Delta_{n, m, 1}}\right|=\left|\Delta_{n, m, 1}\right| O(1 / n) .
$$

На основании (29), (34) и (35) будем иметь

$$
f(z)-\Pi_{n, m}(z)=\frac{R_{n, m}(z)}{Q_{m, n}(z)}=\frac{\Delta_{n, m, 1} z^{n+m+1}(1+O(1 / n))}{\Delta_{n, m}(1+O(1 / n))} .
$$

Теперь для доказательства теоремы 1 достаточно воспользоваться равенствами (12) и (30), которые получены в леммах 1,3 . Теорема 1 доказана.

ДоКАЗАТЕЛЬСТВо ТЕОРЕМЫ 2. Из (5) очевидньм образом вытекает, что

$$
\mathbf{R}_{n, m}(f) \leqslant \frac{\left|\lambda_{1}\right|^{n+m+1} m !}{n^{2 m}(n+1) !}(1+O(1 / n)) .
$$

Для получения оценки снизу для $\mathbf{R}_{n, m}(f)$ воспользуемся леммой В.К. Дзядыка [21, лемма 3.1]. Пусть $r_{n, m}(z)$ - рациональная дробь наилучшего приближения функции $f(z)$, а $\varphi(z)=f(z)-\Pi_{n, m}(z)$. Тогда, учитывая что $\varphi(z)$ имеет не менее $(n+m+1)$-го нуля в круге $|z|<1$, получим

$$
\begin{aligned}
\mathbf{R}_{n, m}(f) & =\left\|f-r_{n, m}\right\|=\left\|f-\Pi_{n, m}-\left(r_{n, m}-\Pi_{n, m}\right)\right\| \\
& =\left\|\varphi-\widetilde{r}_{n+m, 2 m}\right\| \geqslant \mathbf{R}_{n+m, 2 m}(\varphi) \geqslant \min _{|z|=1}|\varphi(z)| .
\end{aligned}
$$

Учитьвая (5) и (36), равенство (6), а вместе с ним и теорема 2 доказаны. 


\section{§4. Доказательства теорем $3,4,5$}

Доказательство теоремы 4 проводится аналогично доказательству теоремы $2 \mathrm{c}$ помощью леммы В.К. Дзядыка и равенства (7), полученного в теореме 3. Поэтому перейдем к доказательству теоремы 3.

ДокаЗАТЕЛЬСТво ТеоРемЫ 3. Пусть $\lambda_{j}=R \exp \left\{\left(\varphi_{0}+2 \pi(j-1) / s\right) i\right\}$, $j=1,2, \ldots, s ;$ a $f(z)=\sum_{j=1}^{s} e^{\lambda_{j} z}$. Тогда для коэффициентов Тейлора $f(z)$ получим представление

$$
c_{n}=\frac{\left(R e^{i \varphi_{0}}\right)^{n}}{n !} \sum_{j=1}^{s} e^{\frac{2 \pi(j-1)}{s} n i}
$$

Следовательно,

$$
\begin{gathered}
c_{n s}=\frac{s\left(R e^{i \varphi_{0}}\right)^{n s}}{(n s) !}, \quad n=0,1, \ldots, \\
c_{j}=0, \quad j \neq n s .
\end{gathered}
$$

Поэтому функция $f(z)$ разлагается в степенной ряд вида

$$
f(z)=\sum_{n=0}^{\infty} c_{n s} z^{n s}
$$

Произведя замену $\xi=z^{s}$, определим функцию $\widetilde{f}(\xi)$ :

$$
\widetilde{f}(\xi)=\sum_{n=0}^{\infty} \widetilde{c}_{n} z^{n}
$$

где $\widetilde{c}_{n}=c_{n s}, n=0,1,2, \ldots$ Очевидно, что

$$
\begin{aligned}
\Pi_{n s, m s}(z ; f) & =\Pi_{n, m}\left(z^{s} ; \widetilde{f}\right) \\
f(z)-\Pi_{n s, m s}(z ; f) & =\widetilde{f}(\xi)-\Pi_{n, m}(\xi ; \widetilde{f}) .
\end{aligned}
$$

Поэтому достаточно изучить поведение аппроксимаций Паде $\Pi_{n, m}(\xi ; \widetilde{f})$. Обозначим через $\widetilde{\Delta}_{n, m}$ определители Адамара функции $\widetilde{f}(\xi)$.

ЛЕмма 4. При указанном выборе чисел $\left(\lambda_{j}\right)_{j=1}^{s}$ для любого фиксированного m и $n \rightarrow \infty$ имеет место асимптотическое равенство

$$
\widetilde{\Delta}_{n, m}=\frac{\left(-s^{2} R^{s} e^{i \varphi_{0} s}\right)^{m(m-1) / 2}}{(n s)^{(s+1) m(m-1) / 2}} \prod_{j=1}^{m}(m-j) ! c_{(n-m+1) s}(1+O(1 / n)) .
$$


ДОКАЗАТЕЛЬСТВО. Из определения $\widetilde{c}_{n}$ и $(37)$ следует, что

$$
\frac{\widetilde{c}_{n+1}}{\widetilde{c}_{n}}=\frac{\left(R e^{i \varphi_{0}}\right)^{s}}{(n s+1)(n s+2) \cdots(n s+s)} .
$$

Тогда, разделив $j$-столбец определителя $\widetilde{\Delta}_{n, m}$ на $\widetilde{c}_{n-m+j}(j=1,2, \ldots, m)$, получим

$$
\begin{aligned}
& \widetilde{\Delta}_{n, m}=z_{1}^{m(m-1) / 2} \prod_{j=1}^{m} \widetilde{c}_{n-m+j} \\
& \times\left|\begin{array}{crcc}
1 & 1 & \ldots & 1 \\
\widetilde{l}_{1}(n-m+1) & \widetilde{l}_{1}(n-m+2) & \ldots & \widetilde{l}_{1}(n) \\
\widetilde{l}_{2}(n-m+1) & \widetilde{l}_{2}(n-m+2) & \ldots & \widetilde{l}_{2}(n) \\
\ldots \ldots \ldots \ldots \ldots \ldots \ldots \ldots \ldots \ldots \ldots \ldots \ldots \ldots \ldots \ldots \ldots \ldots \ldots \ldots \ldots \ldots & \ldots \ldots \ldots
\end{array}\right|
\end{aligned}
$$

где $\widetilde{l}_{i}(j)=\frac{\widetilde{c}_{j+i}}{z_{1}^{i} \widetilde{c}_{j}}, z_{1}=\left(R e^{i \varphi_{0}}\right)^{s}$, a $1 \leqslant i \leqslant m-1, n-m+1 \leqslant j \leqslant n$. С учетом при тех же $i$ и $j$ имеем

$$
\frac{\widetilde{c}_{j+i}}{\widetilde{c}_{j}}=\frac{z_{1}^{i}}{(j s+1)(j s+2) \cdots(j s+i s)} .
$$

Отсюда

$$
\widetilde{\Delta}_{n, m}=z_{1}^{m(m-1) / 2} \widetilde{H}(n, m) \prod_{j=1}^{m} \widetilde{c}_{n-m+j},
$$

где

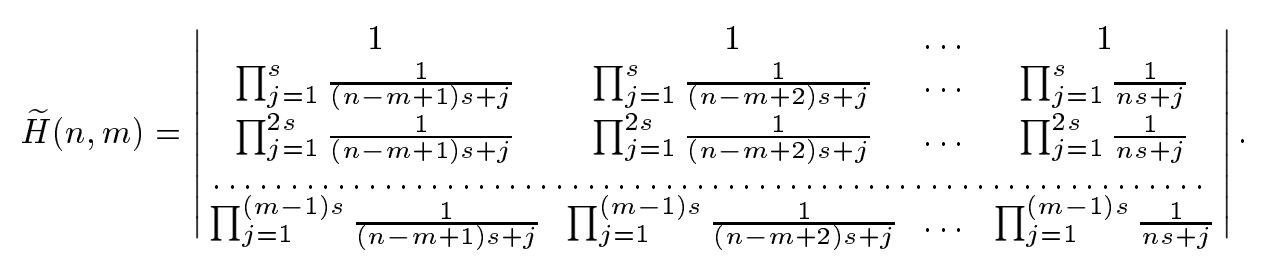

Остается найти асимптотику определителя $\widetilde{H}(n, m)$. Для этого введем в рассмотрение $m-1$ функций

$$
\varphi_{k}(x)=\prod_{j=1}^{k s} \frac{1}{x+j}, \quad k=1,2, \ldots, m-1
$$

и положим $n_{j}=(n-m+j) s, j=1,2, \ldots, m$. Тогда согласно обобшению теоремы Ролля на систему функций $[22$, с. 64$]$, существуют числа $\xi_{j}, j=1,2, \ldots, m, n_{1} \leqslant$ 
$\xi_{j} \leqslant n_{m}$, такие, что

$$
\begin{aligned}
& \widetilde{H}(n, m)=\left|\begin{array}{cccc}
1 & 1 & \ldots & 1 \\
\varphi_{1}\left(n_{1}\right) & \varphi_{1}\left(n_{2}\right) & \ldots & \varphi_{1}\left(n_{m}\right) \\
\varphi_{2}\left(n_{1}\right) & \varphi_{2}\left(n_{2}\right) & \ldots & \varphi_{2}\left(n_{m}\right) \\
\ldots \ldots \ldots \ldots \ldots \ldots \ldots \ldots \ldots \ldots \ldots & \ldots \ldots \ldots \ldots \ldots
\end{array}\right| \\
& =\frac{\prod_{1 \leqslant i<j \leqslant m}\left(n_{j}-n_{i}\right)}{\prod_{j=1}^{m-1} j !}\left|\begin{array}{cccc}
1 & 0 & \ldots & 0 \\
\varphi_{1}\left(\xi_{1}\right) & \varphi_{1}^{\prime}\left(\xi_{2}\right) & \ldots & \varphi_{1}^{(m-1)}\left(\xi_{m}\right) \\
\varphi_{2}\left(\xi_{1}\right) & \varphi_{2}^{\prime}\left(\xi_{2}\right) & \ldots & \varphi_{2}^{(m-1)}\left(\xi_{m}\right) \\
\ldots \ldots \ldots \ldots \ldots \ldots \ldots \ldots \ldots & \ldots \ldots \ldots \ldots
\end{array}\right| \\
& =\frac{\prod_{1 \leqslant i<j \leqslant m}\left(n_{j}-n_{i}\right)}{\prod_{j=1}^{m-1} j !} J_{n, m},
\end{aligned}
$$

где $J_{n, m}$ - алгебраическое дополнение 1 , стоящей в первой строке предыдушего определителя. Используя формулу Лейбница

$$
\left(\prod_{j=1}^{p} \psi_{j}(x)\right)^{(l)}=\sum_{l_{1}+l_{2}+\cdots+l_{p}=l} \frac{l !}{\prod_{j=1}^{p}\left(l_{j}\right) !} \prod_{j=1}^{p} \psi_{j}^{\left(l_{j}\right)}(x)
$$

при $k=1,2, \ldots, m-1, l=1,2, \ldots, m-1$, будем иметь

$$
\begin{aligned}
\left(\varphi_{k}\left(\xi_{l+1}\right)\right)^{(l)} & =\left(\prod_{j=1}^{k s} \frac{1}{x+j}\right)_{\mid x=\xi_{l+1}}^{(l)} \\
& =\sum_{l_{1}+l_{2}+\cdots+l_{k s}=l} \frac{l !}{\prod_{j=1}^{k s}\left(l_{j}\right) !} \prod_{j=1}^{k s}\left(\frac{1}{x}\right)_{\mid x=n s}^{\left(l_{j}\right)}(1+O(1 / n)) \\
& =\left(\frac{1}{x^{k s}}\right)_{\mid x=n s}^{(l)}(1+O(1 / n))=\frac{(-1)^{l}(k s)_{(l)}}{(n s)^{k s+l}}(1+O(1 / n)) .
\end{aligned}
$$

Тогда $J_{n, m}$ можно переписать в виде

$$
\begin{aligned}
& J_{n, m}=
\end{aligned}
$$

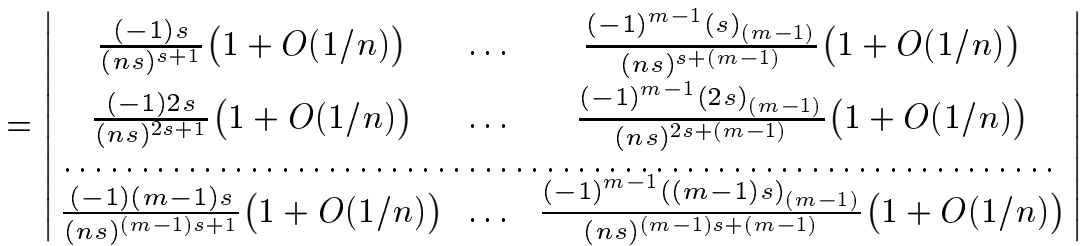


Определитель, составленный из первых слагаемых предыдушего определителя, легко вычисляется:

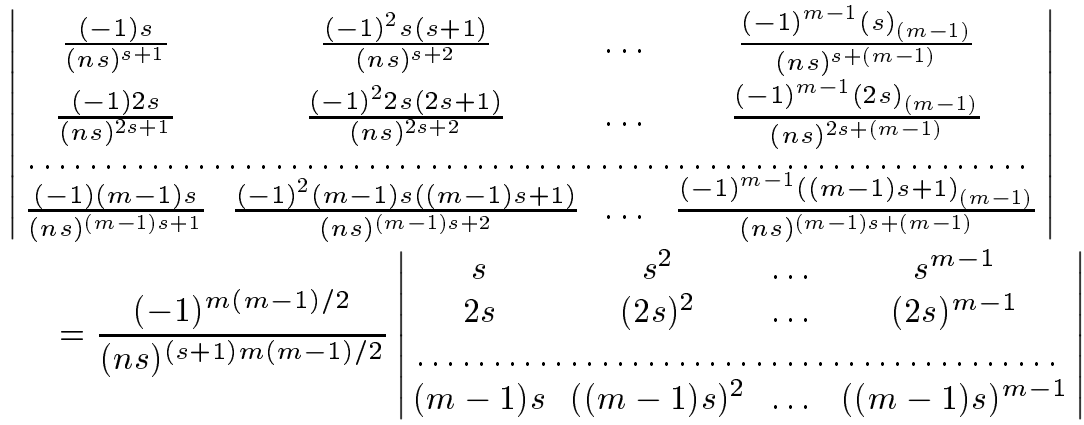

$$
\begin{aligned}
& =\frac{(-1)^{m(m-1) / 2}}{(n s)^{(s+1) m(m-1) / 2}} s^{m(m-1) / 2} \prod_{j=1}^{m-1} j ! \text {. }
\end{aligned}
$$

Вычисленный определитель имеет порядок убывания равный $1 / n^{(s+1) m(m-1) / 2}$. С другой стороны, определитель $J_{n, m}$ представляет собой сумму конечного числа слагаемых, каждое из которых, если оно не содержит множителя $O(1 / n)$, как нетрудно убедиться, имеет такой же порядок убывания $1 / n^{(s+1) m(m-1) / 2}$. Присутствие в слагаемом множителей вида $O(1 / n)$ увеличивает его порядок убьвания по крайней мере на 1 . Поэтому главный член асимптотики определителя $J_{n, m}$ в точности равен вычисленному определителю. Тогда из (43)-(45) и (40) очевидньм образом следует (38). Лемма доказана.

По аналогии введем в рассмотрение определители $\widetilde{\Delta}_{n, m}^{j}, j=1,2, \ldots, m ; \widetilde{\Delta}_{n, m}^{0}=$ $\widetilde{\Delta}_{n, m}$. Рассуждая как и при доказательстве леммы 4 , получим, что

$$
\widetilde{\Delta}_{n, m}^{j}=\widetilde{\Delta}_{n, m} O(1 / n)
$$

Доказательство теоремы 3. таким образом, завершает следуюшая лемма.

ЛЕмма 5. При указанном выборе чисел $\left(\lambda_{j}\right)_{j=1}^{s}$ для любого фиксированного m и $n \rightarrow \infty$ имеет место асимптотическое равенство

$$
\begin{aligned}
\widetilde{\Delta}_{n, m, k}= & \frac{\left(-s^{2} R^{s} e^{i \varphi_{0} s}\right)^{m(m+1) / 2}\left(R^{s} e^{i \varphi_{0} s}\right)^{k-1}}{(n s)^{(s+1) m(m+1) / 2+(k-1) s}} \\
& \times \prod_{j=1}^{m}(m-j) !(j+k-1) \prod_{j=1}^{m+1} c_{(n-m+j) s}(1+O(1 / n)) .
\end{aligned}
$$

ДокАЗАТЕльство. Проделав те же преобразования с определителем $\widetilde{\Delta}_{n, m, k}$, что и в лемме 4 , получим

$$
\widetilde{\Delta}_{n, m, k}=z_{1}^{\frac{m(m+1)}{2}+k-1} \widetilde{H}(n, m, k) \prod_{j=1}^{m+1} \widetilde{c}_{n-m+j},
$$


где

$$
\begin{aligned}
& \tilde{H}(n, m, k)= \\
& =\left|\begin{array}{ccccc}
\prod_{j=1}^{s} \frac{1}{(n-m+1) s+j} & \ldots & \prod_{j=1}^{s} \frac{1}{n s+j} & \prod_{j=1}^{s} \frac{1}{(n+1) s+j} \\
\prod_{j=1}^{2 s} \frac{1}{(n-m+1) s+j} & \ldots & \prod_{j=1}^{2 s} \frac{1}{n s+j} & \prod_{j=1}^{2 s} \frac{1}{(n+1) s+j} \\
\ldots \ldots \ldots \ldots \ldots \ldots \ldots \ldots \ldots \ldots \ldots \ldots \ldots \ldots \ldots \ldots \\
\prod_{j=1}^{(m-1) s} \frac{1}{(n-m+1) s+j} & \ldots & \prod_{j=1}^{(m-1) s} \frac{1}{n s+j} & \prod_{j=1}^{(m-1) s} \frac{1}{(n+1) s+j} \\
\prod_{j=1}^{(m+k-1) s} \frac{1}{(n-m+1) s+j} & \ldots & \prod_{j=1}^{(m+k-1) s} \frac{1}{n s+j} & \prod_{j=1}^{(m+k-1) s} \frac{1}{(n+1) s+j}
\end{array}\right| .
\end{aligned}
$$

Введя обозначения $\varphi_{m}(x)=\prod_{j=1}^{(m+k-1) s} 1 /(x+j), n_{m+1}=(n+1) s$ и применив к системе функций $\left(\varphi_{k}(x)\right)_{k=1}^{m}$ обобщенную теорему Ролля, получим равенство

$$
\widetilde{H}(n, m, k)=\frac{\prod_{1 \leqslant i<j \leqslant m+1}\left(n_{j}-n_{i}\right)}{\prod_{j=1}^{m} j !} J_{n, m, k},
$$

в котором асимптотика определителя $J_{n, m, k}$ находится как и в лемме 4 для определителя $J_{n, m}$. Приведем заключительные вькладки

$$
\begin{aligned}
& J_{n, m, k}=\frac{(-1)^{m(m+1) / 2}}{(n s)^{(s+1) m(m+1) / 2+(k-1) s}}(1+O(1 / n))
\end{aligned}
$$

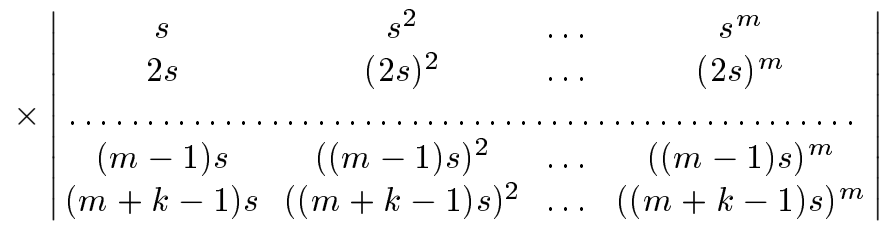

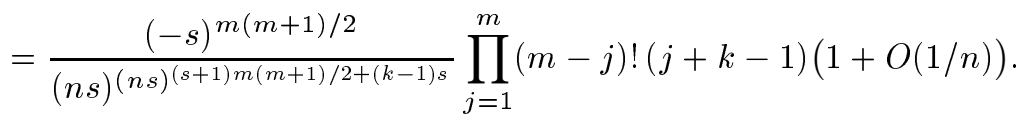

Лемма доказана.

Из лемм 3 и 4, рассуждая так же, как и при доказательстве теоремы 1 , получим

$$
f(z)-\Pi_{n s, m s}(z)=\frac{\widetilde{\Delta}_{n, m, 1}}{\widetilde{\Delta}_{n, m}} z^{(n+m+1) s}(1+O(1 / n)) .
$$

Отсюда следует (7). Теорема 3 доказана.

ДОКАЗАТЕЛЬСТво ТЕОРЕмЫ 5 . Пусть $f(z)$ аналитична в $G$ и задается степенньгм рядом (10). Через $\varkappa(z)$ будем обозначать произведение Бляшке, имеющее $(n-m)$-кратный нуль при $z=0$ и нули в точках $\left(w_{k}\right)_{k=1}^{m},\left|w_{k}\right|<1, k=1,2, \ldots, m$; т.e.

$$
\varkappa(z)=z^{n-m} \prod_{k=1}^{m} \frac{z-w_{k}}{1-\bar{w}_{k} z}=z^{n-m} \frac{z^{m}+\bar{b}_{1} z^{m-1}+\cdots+\bar{b}_{m}}{1+b_{1} z+\cdots+b_{m} z^{m}} .
$$


Введем в рассмотрение частную сумму Фурье функции $f(z)$ по ортогональной системе Такенака-Малькмиста (подробнее см. [23], [24]):

$$
S_{n, m}(z ; f)=\frac{1}{2 \pi i} \int_{|\xi|=1} f(\xi) \frac{\xi \varkappa(\xi)-z \varkappa(z)}{(\xi-z) \xi \varkappa(\xi)} d \xi
$$

Известно, что $S_{n, m}(z ; f)$ есть рациональная функция, имеющая в числителе полином степени не вьшше $n$, а в знаменателе полином $1+b_{1} z+\cdots+b_{m} z^{m}$, интерполируюшая функцию $f(z)$ в корнях произведения Бляшке (47) с кратностью равной кратности корней. Полюса $S_{n, m}(z ; f)$, таким образом, совпадают с точками $\left(1 / \bar{w}_{k}\right)_{k=1}^{m}$ и являются свободными параметрами, которые можно выбирать в зависимости от свойств функции $f(z)$. В [20] доказано, что если $\Delta_{n, m} \neq 0$ и числа $\left(b_{k}\right)_{k=1}^{m}$ выбирать как решение системы уравнений

$$
\sum_{k=1}^{m} b_{k} c_{n+j-k}=-c_{n+j} \quad(j=1,2, \ldots, m),
$$

то знаменатели $S_{n, m}(z ; f)$ и $\Pi_{n, m}(z ; f)$ совпадают и для любых $z \in \bar{D}$

$$
\left|f(z)-S_{n, m}(z ; f)\right| \leqslant c \sum_{j=m+1}^{\infty}\left|\sum_{k=0}^{m} b_{k} c_{n+j-k}\right| .
$$

В условиях теоремы 5 из результатов работы [16] следует, что $\left|b_{k}\right|=O\left(1 / n^{k}\right)$ $(k=1,2, \ldots, m)$. Учитывая, что для суммы экспонент (4)

$$
\left|c_{n+j}\right|=O(1 /(n+j-k) !)
$$

оценивая правую часть последнего неравенства, получим (9). Теорема доказана.

\section{Список литературы}

1. Padé $H$. Memoire sur les développements en fractions continues de la fonction exponentielle // Ann. Sci. Ecole Norm. Super. 1899. V. 16. № 3. P. 394-426.

2. Padé $H$. Sur la représentation approchée d'une fonction par des fractions rationnelles // Ann. Sci. Ecole Norm. Super. 1892. V. 9. P. 1-93.

3. Perron O. Die Lehre von den Kettenbrüchen. New York: Chel. Publ. Comp., 1948.

4. Luke Y.L. The special functions and their approximations. V. 53. New York-London: Acad. Press, 1969.

5. Дзядык В. К., Филозоф Л.И. О скорости сходимости аппроксимаций Паде для некоторых элементарных функций // Матем. сб. 1978. Т. 107(149). С. 347-363.

6. Saff E. B. The convergence of rational functions of best approximation to the exponential function // Trans. Amer. Math. Soc. 1971. V. 153. P. 483-493.

7. Saff E. B. On the degree of best rational approximation to the exponential function // J. Approx. Theory. 1973. V. 9. № 2. P. 97-101.

8. Saff E. B. The covergence of rational functions of best approximation to the exponential function // Proc. Amer. Math. Soc. 1972. V. 32. № 1. P. 187-194.

9. Бейкер Джс., Грейвс-Моррис П. Аппроксимации Паде. М.: Мир, 1986.

10. Гончар A.A., Рахманов E. A. Равновесные распределения и скорость рациональной аппроксимации аналитических функций // Матем. сб. 1987. Т. 134(176). С. 306-352. 
11. Varga R. S. Topics in polynomial and rational interpolation and approximation. University of Montreal Press, 1982.

12. Varga R.S. Scientific computation on some mathematical conjectures // Approximation theory / ed. S. K. Chui, L. L. Schumaker and J. D. Ward Academic Press, 1986. P. 191-209.

13. Trefethen L. N., Gutknecht $M$. The Caratheodory-Fejér method for real rational approximation // SIAM J. Numer. Anal. 1983. V. 20. P. 420-436.

14. Braess D. On the conjecture of Meinardus on rational approximation of $e^{x} / /$ J. Approx. Theory. 1984. V. 40. № 4. P. 375-379.

15. Trefethen $L . N$. The asymptotic accuracy of rational best approximations to $e^{z}$ on a disk // J. Approx. Theory. 1984. V. 40. № 4. P. 380-384.

16. Аптекарев А.И. Асимптотика определителей Адамара и сходимость строк аппроксимаций Паде для суммы экспонент // Матем. сб. 1980. Т. 113(155). С. 520-537.

17. Гончар A. A. О сходимости обобщенных аппроксимаций Паде мероморфных функций // Матем. сб. 1975. Т. 98(140). С. 564-577.

18. Старовойтов А. П., Старовойтова Н. А. Поведение строк таблиц Паде и Чебышёва для суммы экспонент // Докл. Академии наук Беларуси. 1992. Т. 36. № 3-4. С. 202-204.

19. Березкина Л. Л., Русак В.Н. О наилучшей рациональной аптроксимации некоторых целых функций // Весці АН БССР. Сер. фіз.-матем. навук. 1990. № 4. С. 27-32.

20. Русак B. H. Исследование строк рациональной таблицы Чебьшёва для индивидуальных аналитических функций // Весці АН БССР. Сер. фіз.-матем. навук. 1988. №6. С. 26-30.

21. Дзядык B. K. Об асимптотике диагональных аппроксимаций Паде функций $\sin z, \cos z$, $\operatorname{sh} z$ и сh $z$ // Матем. сб. 1979. Т. 108 (150). №2. С. 247-267.

22. Полиа Г., Сеге Г. Задачи и теоремы из анализа. Ч. 2. М.: Наука, 1978.

23. Джрбашян M. М. К теории рядов Фурье по рационалњным функциям // Изв. АН Арм. ССР. Сер. физ.-матем. наук. 1957. Т. 9. № 7. С. 5-83.

24. Русак В. Н. Рациональные функции как аппарат приближения. Минск: Изд-во БГУ, 1979.

Гомельский государственный университет

Поступила в редакцию

30.03 .1995 